\title{
Enriched environment prevents oxidative stress in zebrafish submitted to unpredictable chronic stress
}

\author{
Matheus Marcon ${ }^{1}$, Ricieri Mocelin ${ }^{1}$, Adrieli Sachett ${ }^{1}$, Anna M Siebel ${ }^{2}$, Ana P Herrmann ${ }^{3}$, Angelo Piato \\ Corresp. $1,3,4$ \\ 1 Programa de Pós-graduação em Neurociências, Universidade Federal do Rio Grande do Sul, Porto Alegre, RS, Brazil \\ 2 Programa de Pós-graduação em Ciências Ambientais, Universidade Comunitária da Região de Chapecó, Chapecó, SC, Brazil \\ 3 Programa de Pós-graduação em Farmacologia e Terapêutica, Universidade Federal do Rio Grande do Sul, Porto Alegre, RS, Brazil \\ 4 Zebrafish Neuroscience Research Consortium (ZNRC), Los Angeles, United States \\ Corresponding Author: Angelo Piato \\ Email address: angelopiato@ufrgs.br
}

Background: The enriched environment (EE) is a laboratory housing model that emerged from efforts to minimize the impact of environmental conditions on laboratory animals. Recently, we showed that EE promoted positive effects on behavior and cortisol levels in zebrafish submitted to the unpredictable chronic stress (UCS) protocol. Here, we expanded the characterization of the effects of UCS protocol by assessing parameters of oxidative status in the zebrafish brain and reveal that EE protects against the oxidative stress induced by chronic stress. Methods: Zebrafish were exposed to EE (21 or 28 days) or standard housing conditions and subjected to the UCS protocol for 7 days. Oxidative stress parameters (lipid peroxidation (TBARS), reactive oxygen species (ROS) levels, non-protein thiol (NPSH) and total thiol (SH) levels, superoxide dismutase (SOD) and catalase (CAT) activities were measured in brain homogenate. Results: Our results revealed that UCS increased lipid peroxidation and ROS levels, while decreased NPSH levels and SOD activity, suggesting oxidative damage. EE for 28 days prevented all changes induced by the UCS protocol, and EE for 21 days prevented the alterations on NPSH levels, lipid peroxidation and ROS levels. Both EE for 21 or 28 days increased CAT activity. Discussion: Our findings reinforce the idea that EE exerts neuromodulatory effects in the zebrafish brain. EE promoted positive effects as it helped maintain the redox homeostasis, which may reduce the susceptibility to stress and its oxidative impact. 
1 Enriched environment prevents oxidative stress in zebrafish submitted to unpredictable

3

4 Matheus Marcon¹, Ricieri Mocelin ${ }^{1}$, Adrieli Sachett ${ }^{1}$, Anna M. Siebel ${ }^{2}$, Ana P. Herrmann ${ }^{1}$, Angelo

5 Piato $^{1,2,3 *}$

$6 \quad{ }^{1}$ Programa de Pós-graduação em Neurociências, ICBS, Universidade Federal do Rio Grande do

7 Sul, Porto Alegre, Rio Grande do Sul, Brazil.

82 Programa de Pós-graduação em Ciências Ambientais, Universidade Comunitária da Região de

9 Chapecó, Chapecó, SC, Brazil.

$10{ }^{3}$ Zebrafish Neuroscience Research Consortium (ZNRC), Slidell, LA, USA.

11

$12 *$ Correspondence to:

13 Angelo Piato, Ph.D.

14 Programa de Pós-Graduação em Neurociências, ICBS, Universidade Federal do Rio Grande do Sul.

15 Av. Sarmento Leite 500/305, Porto Alegre, RS, Brazil.

16 90050-170; Phone/Fax: +55 51 33083121; E-mail address: angelopiato@ufrgs.br 


\section{ABSTRACT}

36

Background: The enriched environment (EE) is a laboratory housing model that emerged from efforts to minimize the impact of environmental conditions on laboratory animals. Recently, we showed that EE promoted positive effects on behavior and cortisol levels in zebrafish submitted to the unpredictable chronic stress (UCS) protocol. Here, we expanded the characterization of the effects of UCS protocol by assessing parameters of oxidative status in the zebrafish brain and reveal that EE protects against the oxidative stress induced by chronic stress.

Methods: Zebrafish were exposed to EE (21 or 28 days) or standard housing conditions and subjected to the UCS protocol for 7 days. Oxidative stress parameters (lipid peroxidation (TBARS), reactive oxygen species (ROS) levels, non-protein thiol (NPSH) and total thiol (SH) levels, superoxide dismutase (SOD) and catalase (CAT) activities were measured in brain homogenate.

Results: Our results revealed that UCS increased lipid peroxidation and ROS levels, while decreased NPSH levels and SOD activity, suggesting oxidative damage. EE for 28 days prevented all changes induced by the UCS protocol, and EE for 21 days prevented the alterations on NPSH levels, lipid peroxidation and ROS levels. Both EE for 21 or 28 days increased CAT activity.

Discussion: Our findings reinforce the idea that EE exerts neuromodulatory effects in the zebrafish brain. EE promoted positive effects as it helped maintain the redox homeostasis, which may reduce the susceptibility to stress and its oxidative impact. 


\section{INTRODUCTION}

67 acknowledged in scientific discussions (Kempermann, Kuhn \& Gage, 1997; Animals, 2011; Kim et al., 2017). Sherwin (2004) for example, reviewed the effects of standard laboratory cages design and husbandry in rodents. The author argues that validity of research data is another reason to improve housing conditions of experimental animals, besides the more often mentioned welfare aspect. Different husbandry conditions could contribute to the observed data variability and irreproducibility among different laboratories. For zebrafish, however, studies comparing the impact of housing conditions on research outcomes are scarce. The standard laboratory condition for this species consists of housing the animal in shoals in barren tanks only with a recirculation system, heater thermostat (temperature control), and water in ideal conditions including physical, chemical and biological characteristics $\mathrm{pH}$, salinity, alkalinity, hardness, dissolved oxygen and nitrogen residues) (Lawrence \& Mason, 2012). However, this housing environment is very far from the natural habitat conditions to zebrafish, that lives in shallow water with aquatic vegetation and gravel substrates (Arunachalam et al., 2013).

In this context, the practice of enriched environment (EE) arose from efforts to minimize the impact of environmental conditions on laboratory animals (Diamond, Krech \& Rosenzweig, 1964; Kempermann, Kuhn \& Gage, 1997; Young et al., 1999; van Praag, Kempermann \& Gage, 2000; Bennett et al., 2006). EE is a laboratory housing model that aims to approximate the housing condition to the natural habitat of the animals. This form of housing includes interventions that contributes to increase stimulation of sensory, motor and cognitive neuronal systems of the brain, and it allows or facilitates the animals to develop natural and species-specific behaviors (van Praag,

89 Kempermann \& Gage, 2000; Lazarov et al., 2005; Meshi et al., 2006; Nithianantharajah \& Hannan, 90 2006; Tanti et al., 2013).

A growing body of evidence reports the beneficial effects of EE for several species of 92 laboratory animals (Nilsson et al., 1999; Brown et al., 2003; Sale et al., 2007; Sztainberg \& Chen, 93 2010; Toth et al., 2011; Gapp et al., 2016). Studies in rodents report that EE has positive effects on 
94 the maintenance of the redox state, promoting protection against oxidative stress. For example, it

95 was demonstrated that rats housed in EE presented reduced oxidative stress biomarkers, such as

96 thiobarbituric acid reactive substances (TBARS), protein oxidation, superoxide anion $\left(\mathrm{O}_{2}{ }^{\bullet-}\right)$ activity

97 and higher values for antioxidant parameters, such as the total radical antioxidant parameter,

98 catalase (CAT) and superoxide dismutase (SOD) when compared to animals housed in standard

99 laboratory conditions (Mármol et al., 2015). A report showed that EE attenuated the upregulation of

100 biomarkers of ROS production, such as levels of oxidase 2 (NOX2) and 8-hydroxy-2-

101 deoxyguanosine (8-OH-dG) induced by a rat model of post-traumatic stress disorder (Sun et al.,

102 2016). In addition, EE promoted neuroprotection through epigenetic mechanisms because it

103 increased levels of DNA methylation and reduced levels of hydroxymethylation, as well as

104 increased histone acetylation levels of $\mathrm{H} 3$ and $\mathrm{H} 4$. This resulted in increased expression of genes

105 encoding oxidative machinery proteins, such as Hmox1, Aox1, and Cox2, and reduced expression

106 of inflammatory genes such as IL-6 E Cxcl10 (Griñan-Ferré et al., 2016) .

107 Lastly, although some evidence suggests promising results for EE on oxidative stress in

108 rodents, studies that report the effects of EE on this parameter in zebrafish are still scarce.

109 Considering that in our previous study we demonstrated that EE prevented the increase of ROS in

110 zebrafish submitted to the unpredictable chronic stress (UCS) protocol (Marcon et al., 2018), we

111 hypothesized that zebrafish submitted to UCS protocol housed in EE would be less vulnerable to

112 oxidative stress. Therefore, in this study we tested the effects of EE in zebrafish submitted to UCS

113 on a range of oxidative stress parameters including lipid peroxidation (TBARS), reactive oxygen

114 species (ROS), non-protein thiol (NPSH) levels, total thiol (SH) levels, superoxide dismutase

115 (SOD) activity, and catalase (CAT) activity.

116

117 MATERIAL AND METHODS

118

119 Animals

120

121

A total of 150 short fin wild-type (WT) adult zebrafish (Danio rerio) 50:50 male/female

122 ratio over 6-month-old were purchased from Delphis aquariums (Porto Alegre, Brazil). The fish

123 were kept in a closed acclimation tank system of $16 \mathrm{~L}(40 \times 20 \times 24 \mathrm{~cm},<2$ fish per liter $)$ for two

124 weeks. Tanks were filled with non-chlorinated tap water, well-aerated in appropriate conditions as 
125 previously reported by Marcon et al. (2018). The illumination of the room was $14 / 10 \mathrm{~h} \mathrm{light/dark}$

126 photoperiod cycle (lights on at 06:00 am). The fish were fed twice a day with a commercial flake

127 fish food (Alcon BASIC $®$, Alcon, Brazil) and nauplii of brine shrimp (Artemia salina). The amount

128 of food was calculated based on the number of fish per tank and followed the instructions of the

129 Zebrafish Book (Westerfield, 2000). All experiments were approved by the Ethics Committee of

130 Universidade Federal do Rio Grande do Sul (\#30992/2015).

131

132 Experimental procedures

133

EE methodology followed that described by Marcon et al. (2018) and is shown in Figure 1

and 2. After the acclimation period (two weeks), zebrafish were randomly assigned to 1 of 2 experimental housing environments: barren tank (BARREN) or enriched environment (EE). BARREN condition consists of standard laboratory tank as described above and containing only water, heater, filter, and aeration system while EE condition consists the same BARREN condition plus tank gravel in the bottom (English sea stones, $4-9 \mathrm{~mm}, 3 \mathrm{~cm}$ high from the bottom of the tank), a ruin-like plastic object, and three submerged plastic plants (two $10 \mathrm{~cm}$ tall and one $20 \mathrm{~cm}$ tall)

(Figure 1). All tanks of both experimental acclimation conditions were kept in a horizontal plane at the same room, so we used a white frosted cardboard $(30 \times 60 \mathrm{~cm})$ placed only in between tanks to prevent visual contact of fish from different tanks.

Zebrafish were kept in barren tank (BARREN) or enriched environment tank for 21 (EE 21) or 28 (EE 28) days. In both housing conditions, in the last seven days of the experimental protocol the animals were again divided into two experimental subgroups (non-stressed or stressed, respectively, $\mathrm{S}$ - and $\mathrm{S}+$ ). The $\mathrm{S}+$ groups were submitted to unpredictable chronic stress (UCS) protocol detailed bellow. At the end of the experimental protocol on day 29th (24 h of the last intervention), the animals were removed from their tanks by using a net and immediately anesthetized by rapid cooling (immersion in water at $2-4^{\circ} \mathrm{C}$ ). After cessation of opercular movements zebrafish were euthanized by decapitation. The brain was used for analysis of oxidative 152 stress. 
156 To induce stress in the zebrafish a UCS protocol was used which is already well established 157 and described in our previous studies (Piato et al., 2011; Marcon et al., 2016; Rambo et al., 2017).

158 UCS protocol was based on the following stressors (1) heating tank water up to $33^{\circ} \mathrm{C}(30 \mathrm{~min})$; (2)

159 cooling tank water to $23{ }^{\circ} \mathrm{C}(30 \mathrm{~min})$; (3) crowding of 12 animals in a $300-\mathrm{mL}$ beaker (50 min); (4)

160 transferring the animals to other tank with low water level exposing the dorsal body wall (2 min);

161 (5) tank change, three consecutive times with 30-min interval; and (6) chasing with a net (8 min)

162 that were randomly presented twice a day for 7 consecutive days (day 21 to day 28 ) to the animals

163 of the stressed groups. The non-stressed (S-) animals were maintained in the same room and did not

164 undisturbed throughout the experiments.

165

166

\section{Oxidative stress analysis}

167

168

For brain tissue preparation, immediately after euthanasia, fish were dissected out in ice and to each sample used five brains were pooled that were gently homogenized in ice-cold phosphate buffered saline $\left(\mathrm{PBS}\right.$, Sigma Aldrich ${ }^{\circledR}$ ) $\mathrm{pH} 7.4$ and centrifuged at $10,000 \times \mathrm{g}$ for 5 min at $4{ }^{\circ} \mathrm{C}$ to remove cellular debris. The supernatants were collected and used for estimation biomarkers associated with oxidation mechanisms and induction of oxidative stress described herein.

173

\section{Lipid peroxidation (TBARS)}

Lipid peroxidation (Draper \& Hadley, 1990) was estimated by monitoring thiobarbituric acid reactive substance (TBARS) production. Briefly, a volume equal to 50-70 $\mu$ g protein of brain homogenate were added to $150 \mu \mathrm{L}$ of $2 \%$ trichloroacetic acid (TCA, Sigma Aldrich ${ }^{\circledR}$ ) and centrifuged $(10,000 \times \mathrm{g}, 10 \mathrm{~min})$. The supernatants were collected, mixed with $150 \mu \mathrm{L}$ of $0.5 \%$ thiobarbituric acid (TBA, Sigma Aldrich ${ }^{\circledR}$ ) and then heated at $100{ }^{\circ} \mathrm{C}$ for $30 \mathrm{~min}$. The reading of TBARS levels occurred in the microplate reader in absorbance at $532 \mathrm{~nm}$, using 1,1,3,3tetramethoxypropane (TMP, Sigma Aldrich ${ }^{\circledR}$ ) as a standard. Results were expressed as nanomoles (nmol) $\mathrm{MDA} / \mathrm{mg}$ protein $(\mathrm{n}=5)$. 

fluorescent probe 2',7'-dichlorofluorescin diacetate (DCFH-DA, Sigma Aldrich ${ }^{\circledR}$ ) was used. Briefly, $25 \mu \mathrm{L}$ of brain homogenate was incubated with of $1 \mathrm{mM}$ DCFH-DA and PBS buffer at $37{ }^{\circ} \mathrm{C}$ for 30 min. ROS levels was estimated in the microplate reader in fluorescence at $520 \mathrm{~nm}$ of emission and $480 \mathrm{~nm}$ of excitation using dichlorofluorescein (DCF) as standard. Results were expressed as relative fluorescence unit $(\mathrm{RFU})(\mathrm{n}=5)$.

193

Non-protein thiol (NPSH) levels

To estimate NPSH levels (Ellman, 1959), equal volumes $\left(30_{\mu} \mathrm{L}\right)$ of brain preparation and $6 \%$ trichloroacetic acid $\left(\right.$ Sigma Aldrich $\left.{ }^{\circledR}\right)$ was mixed and centrifuged $\left(3,000 \times \mathrm{g}, 10 \mathrm{~min}\right.$ at $\left.4{ }^{\circ} \mathrm{C}\right)$. Subsequently, an aliquot of supernatant ( $50 \mu \mathrm{g}$ protein) was further mixed with $10 \mathrm{mM}$ 5,5-dithiobis-2-nitrobenzoic acid (DTNB, Sigma Aldrich ${ }^{\circledR}$ ) dissolved in ethanol and the intense yellow color developed was measured in the microplate reader at $412 \mathrm{~nm}$ after $1 \mathrm{~h}$ of incubation at room temperature. Results were expressed as nmol NPSH/mg of protein $(n=5)$.

202

\section{Total thiol (SH) levels}

To estimate SH levels (Ellman, 1959), a volume equal to $50 \mu \mathrm{g}$ protein of brain homogenate was mixed with $10 \mathrm{mM}$ 5,5-dithio-bis-2-nitrobenzoic acid (DTNB, Sigma Aldrich ${ }^{\circledR}$ ) dissolved in ethanol (Sigma Aldrich ${ }^{\circledR}$ ). The intense yellow color developed was measured in the microplate reader at $412 \mathrm{~nm}$ after $1 \mathrm{~h}$ of incubation at room temperature. Results were expressed as nmol $\mathrm{SH} / \mathrm{mg}$ of protein $(\mathrm{n}=5)$.

\section{Superoxide dismutase (SOD) activity}

SOD activity (Misra \& Fridovich, 1972) was estimated by quantifying the inhibition of

214 superoxide-dependent adrenaline auto-oxidation. Adrenochrome formation rate was observed at 480

$215 \mathrm{~nm}$ in the microplate reader in a reaction medium containing glycine- $\mathrm{NaOH}(50 \mathrm{mM}, \mathrm{pH} 10$, Sigma

216 Aldrich $\left.^{\circledR}\right)$, epinephrine (60 mM, pH 1.7, Sigma Aldrich $\left.{ }^{\circledR}\right)$, and homogenate brain (15-30-60 $\mu \mathrm{g}$ of

217 protein). Results were expressed in Units/mg protein $(n=5)$. 


\section{Catalase (CAT) activity}

220

CAT activity (Aebi, 1984) was estimated by measuring the rate of decrease in hydrogen

222 peroxide $\left(\mathrm{H}_{2} \mathrm{O}_{2}\right)$ absorbance at $240 \mathrm{~nm}$. Assay mixture consisted of potassium phosphate buffer

223 (Sigma Aldrich $\left.^{\circledR}\right), \mathrm{H}_{2} \mathrm{O}_{2}\left(1 \mathrm{M}\right.$, Sigma Aldrich $\left.{ }^{\circledR}\right)$ and brain homogenate $(30 \mu \mathrm{g}$ protein). The results

224 were expressed in Units/mg protein $(n=5)$.

225

226

\section{Protein determination}

227

228

Protein was determined by the Coomassie blue method (Bradford, 1976) using bovine serum albumin (Sigma Aldrich ${ }^{\circledR}$ ) as standard. Absorbance of samples was measured at $595 \mathrm{~nm}$.

\section{Statistical analysis}

232

Kolmogorov-Smirnov and Levene tests were used to determine the normal distribution of

234 the data and homogeneity of variance, respectively. Results were analyzed by two-way ANOVA

235 (stress and enriched environment as independent factors) followed by Tukey post hoc test for

236 comparisons within groups and between housing conditions. Differences were considered

237 significant at $\mathrm{p}<0.05$. The data were expressed as a mean + standard error of the mean (S.E.M.).

238

\section{RESULTS}

Figure 3 shows the effects of EE on biochemical parameters associated with oxidative stress

242 (TBARS and ROS) in zebrafish submitted to UCS and summarizes the two-way ANOVA analyzes.

243 Regarding TBARS (Figure 3A), two-way ANOVA revealed that UCS interacted with EE: stress

244 only increased TBARS levels when fish were housed in barren tanks, but not when they were

245 housed for 21 or 28 days of EE. Regarding ROS (Figure 3B), two-way ANOVA also revealed an

246 interaction between UCS and EE: increased ROS levels were observed only in stressed fish from

247 barren tanks, but not from enriched tanks.

248 Figures 4 and 5 show the effects of EE on biochemical parameters associated with 
249 antioxidant mechanisms (NPSH and SH levels, SOD and CAT activity) in zebrafish submitted to

250 UCS. In Figure 4A, two-way ANOVA revealed an interaction between UCS and EE for NPSH

251 levels: stress decreased NPSH levels only in fish from barren tanks, while EE for 21 or 28 days

252 prevented this effect of stress. Regarding SH levels (Figure 4B), two-way ANOVA revealed an

253 interaction but no main effects of UCS and EE; post hoc tests, however, did not reach significance

254 for multiple comparisons between groups.

255 In Figure 5A, two-way ANOVA for SOD activity revealed an interaction between UCS and

256 EE: stress decreased SOD activity, which was prevented only when fish were housed in EE for 28,

257 but not 21 days. Regarding CAT activity (Figure 5B), two-way ANOVA revealed main effects for

258 UCS and EE, but no interaction between these factors; overall, stress decreased while EE increased

259 CAT activity.

260

261

\section{DISCUSSION}

262

263

In this study, we replicate a previous result and expand the characterization of the effects of

264

265

EE on mechanisms associated with antioxidant defenses and oxidative stress in zebrafish submitted

266

to UCS. We demonstrated for the first time that UCS protocol induced several changes in redox

267 homeostasis in the zebrafish brain and revealed that EE has a protective effect against the oxidative stress induced by the UCS protocol.

UCS protocol induces several biochemical changes in the zebrafish brain and through

269 sustained activation of the neuroendocrine axis leads to increased cortisol levels (Piato et al., 2011; Manuel et al., 2014; Marcon et al., 2016; Rambo et al., 2017; Song et al., 2017). This was confirmed by the results recently published in our previous study, which showed that the UCS protocol increased cortisol levels while EE for 21 or 28 days prevented this increase (Marcon et al., 273 2018). In this way, the response to sustained stress leads to great energy expenditure and for this 274 reason some cellular metabolic processes are accelerated (Otte et al., 2016), such as oxidative 275 phosphorylation (Zorov, Juhaszova \& Sollott, 2014) and $\beta$-oxidation of fatty acids (Carracedo, 276 Cantley \& Pandolfi, 2013). As a consequence, the excessive production of ROS can reach levels

277 above the antioxidant defense capacity of the organism and consequently oxidize cellular structures 278 leading to oxidative stress (Sies, Berndt \& Jones, 2017; Poprac et al., 2017). 
Particular features of nervous tissue, such as neurotransmitters metabolism, high iron 280 content, low antioxidant capacity, neuronal membrane rich in polyunsaturated fatty acids and high 281 oxygen consumption, make the brain an organ extremely susceptible to oxidative stress (Clarke \& 282 Sokoloff, 1999; Halliwell, 2006). Therefore, sustained antioxidant mechanisms are necessary for the 283 maintenance of cerebral homeostasis (Finkel \& Holbrook, 2000).

284 Glutathione (GSH) is the main antioxidant component in brain tissue. It is essential for 285 maintenance of redox homeostasis, serving as the cofactor of the enzymes glutathione peroxidase 286 (GPx) and glutathione-S-transferase (GST) and a direct neutralizer of ROS (Dringen, 2000; Dringen 287 \& Hirrlinger, 2003). Here we showed that the UCS protocol decreased NPSH levels, a measure that 288 reflects the levels of GSH, while it did not alter the SH levels (thiols groups associated with 289 cysteine residues), suggesting that chronic stress promoted the depletion of cerebral GSH in 290 zebrafish.

291 At the same time, the UCS protocol decreased SOD but did not alter CAT activities.

292 Physiologically the SOD enzyme plays a key role in neutralizing the superoxide anion $\left(\mathrm{O}_{2}{ }^{--}\right)$to 293 hydrogen peroxide $\left(\mathrm{H}_{2} \mathrm{O}_{2}\right)$, which is synergistically converted to water $\left(\mathrm{H}_{2} \mathrm{O}\right)$ and oxygen $\left(\mathrm{O}_{2}\right)$ by 294 CAT (Fukai \& Ushio-Fukai, 2011). Therefore, we hypothesized the decrease in the SOD activity 295 induced by UCS may led to an excessive accumulation of $\mathrm{O}_{2}{ }^{\bullet-} \cdot \mathrm{O}_{2}{ }^{\bullet-}$ in high concentrations may 296 contribute to oxidative stress through direct or indirect damage, for example, by the formation of 297 other reactive species, such as peroxynitrite $\left(\mathrm{ONOO}^{-}\right), \mathrm{H}_{2} \mathrm{O}_{2}$ or hydroxyl radical $\left(\mathrm{OH}^{\bullet}\right)$ (Fenton 298 reaction) (Pacher, Beckman \& Liaudet, 2007). Additionally, we revealed here that the UCS protocol 299 increases ROS production and therefore we suggest that the high levels of ROS associated with the 300 decrease of the antioxidant mechanisms (GSH level and decreased SOD activity) led to an 301 imbalance between its production and detoxification and consequently increased lipid peroxidation 302 in stressed animals leading to oxidative stress. This is according to a previous study that showed a

303 decrease in the values of total antioxidant status, SOD activity and the increase of lipid peroxidation 304 in mice submitted to chronic unpredictable mild stress (Biala et al., 2017).

305 Oxidative stress is related to the development of mental disorders (Ng et al., 2008) and it 306 was demonstrated to contribute to the pathophysiology of neurodegenerative diseases (Christen, 307 2000). Therefore, it is remarkable the need for studies that bring new discoveries in this line.

308 Interestingly, here, we have shown for the first time that EE promoted protection against oxidative

309 stress induced by UCS. We report that EE for 28 days prevented all changes induced by UCS in the 
310 oxidative status while EE for 21 days prevented the decreased of NPSH levels and the increased of

311 the lipid peroxidation and ROS levels. Besides, both EE for 21 or 28 days increased the CAT

312 activity. Previously, some studies had already reported the protective potential of EE on

313 maintenance of redox homeostasis. EE showed to prevent DNA oxidation (Kang et al., 2016; Sun et

314 al., 2016), the increase of carbonyl protein (Herring et al., 2008), the increase of total free radicals

315 content (Cechetti et al., 2012) and the increase of lipid peroxidation (Muhammad et al., 2017) in

316 rodents.

317 In this study, we suggest that EE prevented the oxidative by preventing the decrease of

318 antioxidant defenses (GSH level and SOD enzyme activity), as well as the increase of ROS

319 levels. Furthermore, EE increases the expression of glucocorticoid receptors (Shilpa et al., 2017),

320 which is associated with downregulation of neuroendocrine axis activity; this occurs by negative

321 feedback at the cortisol receptor and reduces the response to sustained stress.

322

\section{CONCLUSION}

324

Our findings are in agreement with our previous study and together with the literature findings reinforce the idea that EE exerts neuromodulatory effects. Here, we revealed that EE promoted positive effects in the maintenance of redox homeostasis, which may reduce the susceptibility to stress and its oxidative impact. However, our data are still preliminary and require further investigation to establish and clarify the exact neurobiological mechanisms by which EE prevents changes in oxidative status. Also, we reinforce and suggest that zebrafish is a suitable animal model to investigate the neurobiology of stress and the effects of EE.

\section{REFERÊNCIAS}

Aebi H. 1984. Catalase in vitro. Methods in Enzymology 105:121-126. methylmercury and trimethyltin neurotoxicity. Neurotoxicology 13:637-648.

339 Management. National Academies Press (US).

340 Arunachalam M., Raja M., Vijayakumar C., Malaiammal P., Mayden RL. 2013. Natural history

341 of zebrafish (Danio rerio) in India. Zebrafish 10:1-14. DOI: 10.1089/zeb.2012.0803.

342 Bennett JC., McRae PA., Levy LJ., Frick KM. 2006. Long-term continuous, but not daily,

343 environmental enrichment reduces spatial memory decline in aged male mice. Neurobiology of 
344 Learning and Memory 85:139-152. DOI: 10.1016/j.nlm.2005.09.003.

345 Biala G., Pekala K., Boguszewska-Czubara A., Michalak A., Kruk-Slomka M., Budzynska B.

346 2017. Behavioral and Biochemical Interaction Between Nicotine and Chronic Unpredictable

347 Mild Stress in Mice. Molecular Neurobiology 54:904-921. DOI: 10.1007/s12035-016-9701-0.

348 Bradford MM. 1976. A rapid and sensitive method for the quantitation of microgram quantities

349 of protein utilizing the principle of protein-dye binding. Analytical Biochemistry 72:248-254.

350 Brown J., Cooper-Kuhn CM., Kempermann G., Van Praag H., Winkler J., Gage FH., Kuhn HG.

351 2003. Enriched environment and physical activity stimulate hippocampal but not olfactory bulb

352 neurogenesis. The European Journal of Neuroscience 17:2042-2046.

353 Carracedo A., Cantley LC., Pandolfi PP. 2013. Cancer metabolism: fatty acid oxidation in the

354 limelight. Nature reviews. Cancer 13:227-232. DOI: 10.1038/nrc3483.

355 Cechetti F., Worm PV., Lovatel G., Moysés F., Siqueira IR., Netto CA. 2012. Environmental

356 enrichment prevents behavioral deficits and oxidative stress caused by chronic cerebral

357 hypoperfusion in the rat. Life Sciences 91:29-36. DOI: 10.1016/j.1fs.2012.05.013.

358 Christen Y. 2000. Oxidative stress and Alzheimer disease. The American Journal of Clinical

359 Nutrition 71:621s-629s.

360 Clarke DD., Sokoloff L. 1999. Circulation and Energy Metabolism of the Brain.

361 Diamond MC., Krech D., Rosenzweig MR. 1964. The effectsof an enriched environment on the

362 histology of the rat cerebral cortex. The Journal of Comparative Neurology 123:111-120.

363 Draper HH., Hadley M. 1990. Malondialdehyde determination as index of lipid peroxidation.

364 Methods in Enzymology 186:421-431.

365 Dringen R. 2000. Metabolism and functions of glutathione in brain. Progress in Neurobiology

366 62:649-671.

367 Dringen R., Hirrlinger J. 2003. Glutathione pathways in the brain. Biological Chemistry

368 384:505-516. DOI: 10.1515/BC.2003.059.

369 Ellman GL. 1959. Tissue sulfhydryl groups. Archives of Biochemistry and Biophysics 82:70-77.

370 Finkel T., Holbrook NJ. 2000. Oxidants, oxidative stress and the biology of ageing. Nature

371 408:239-247. DOI: 10.1038/35041687.

372 Fukai T., Ushio-Fukai M. 2011. Superoxide Dismutases: Role in Redox Signaling, Vascular

373 Function, and Diseases. Antioxidants \& Redox Signaling 15:1583-1606. DOI:

374 10.1089/ars.2011.3999.

375 Gapp K., Bohacek J., Grossmann J., Brunner AM., Manuella F., Nanni P., Mansuy IM. 2016.

376 Potential of Environmental Enrichment to Prevent Transgenerational Effects of Paternal Trauma.

377 Neuropsychopharmacology: Official Publication of the American College of

378 Neuropsychopharmacology 41:2749-2758. DOI: 10.1038/npp.2016.87.

379 Griñan-Ferré C., Puigoriol-Illamola D., Palomera-Ávalos V., Pérez-Cáceres D., Companys-

380 Alemany J., Camins A., Ortuño-Sahagún D., Rodrigo MT., Pallàs M. 2016. Environmental

381 Enrichment Modified Epigenetic Mechanisms in SAMP8 Mouse Hippocampus by Reducing

382 Oxidative Stress and Inflammaging and Achieving Neuroprotection. Frontiers in Aging

383 Neuroscience 8. DOI: 10.3389/fnagi.2016.00241.

384 Halliwell B. 2006. Oxidative stress and neurodegeneration: where are we now? Journal of

385 Neurochemistry 97:1634-1658. DOI: 10.1111/j.1471-4159.2006.03907.x.

386 Herring A., Blome M., Ambrée O., Sachser N., Paulus W., Keyvani K. 2008. Reduction of

387 cerebral oxidative stress following environmental enrichment in mice with Alzheimer-like

388 pathology. Brain Pathology (Zurich, Switzerland) 20:166-175. DOI: 10.1111/j.1750-

389 3639.2008.00257.x. 
390 Kang H., Choi D-H., Kim S-K., Lee J., Kim Y-J. 2016. Alteration of Energy Metabolism and

391 Antioxidative Processing in the Hippocampus of Rats Reared in Long-Term Environmental

392 Enrichment. Developmental Neuroscience 38:186-194. DOI: 10.1159/000446772.

393 Kempermann G., Kuhn HG., Gage FH. 1997. More hippocampal neurons in adult mice living in

394 an enriched environment. Nature 386:493-495. DOI: 10.1038/386493a0.

395 Kim WY., Cho BR., Kwak MJ., Kim J-H. 2017. Interaction between trait and housing condition

396 produces differential decision-making toward risk choice in a rat gambling task. Scientific

397 Reports 7:5718. DOI: 10.1038/s41598-017-06408-4.

398 Lawrence C., Mason T. 2012. Zebrafish Housing Systems: A Review of Basic Operating

399 Principles and Considerations for Design and Functionality. ILAR journal / National Research

400 Council, Institute of Laboratory Animal Resources 53:179-191. DOI: 10.1093/ilar.53.2.179.

401 Lazarov O., Robinson J., Tang Y-P., Hairston IS., Korade-Mirnics Z., Lee VM-Y., Hersh LB.,

402 Sapolsky RM., Mirnics K., Sisodia SS. 2005. Environmental enrichment reduces Abeta levels

403 and amyloid deposition in transgenic mice. Cell 120:701-713. DOI: 10.1016/j.cell.2005.01.015.

404 LeBel CP., Ali SF., McKee M., Bondy SC. 1990. Organometal-induced increases in oxygen

405 reactive species: the potential of 2',7'-dichlorofluorescin diacetate as an index of neurotoxic

406 damage. Toxicology and Applied Pharmacology 104:17-24.

407 Manuel R., Gorissen M., Zethof J., Ebbesson LOE., van de Vis H., Flik G., van den Bos R. 2014.

408 Unpredictable chronic stress decreases inhibitory avoidance learning in Tuebingen long-fin

409 zebrafish: stronger effects in the resting phase than in the active phase. The Journal of

410 Experimental Biology 217:3919-3928. DOI: 10.1242/jeb.109736.

411 Marcon M., Herrmann AP., Mocelin R., Rambo CL., Koakoski G., Abreu MS., Conterato

412 GMM., Kist LW., Bogo MR., Zanatta L., Barcellos LJG., Piato AL. 2016. Prevention of

413 unpredictable chronic stress-related phenomena in zebrafish exposed to bromazepam, fluoxetine

414 and nortriptyline. Psychopharmacology. DOI: 10.1007/s00213-016-4408-5.

415 Marcon M., Mocelin R., Benvenutti R., Costa T., Herrmann AP., de Oliveira DL., Koakoski G.,

416 Barcellos LJG., Piato A. 2018. Environmental enrichment modulates the response to chronic

417 stress in zebrafish. The Journal of Experimental Biology. DOI: 10.1242/jeb.176735.

418 Mármol F., Rodríguez CA., Sánchez J., Chamizo VD. 2015. Anti-oxidative effects produced by

419 environmental enrichment in the hippocampus and cerebral cortex of male and female rats. Brain

420 Research 1613:120-129. DOI: 10.1016/j.brainres.2015.04.007.

421 Meshi D., Drew MR., Saxe M., Ansorge MS., David D., Santarelli L., Malapani C., Moore H., 422 Hen R. 2006. Hippocampal neurogenesis is not required for behavioral effects of environmental

423 enrichment. Nature Neuroscience 9:729-731. DOI: 10.1038/nn1696.

424 Misra HP., Fridovich I. 1972. The role of superoxide anion in the autoxidation of epinephrine

425 and a simple assay for superoxide dismutase. The Journal of Biological Chemistry 247:3170-

4263175.

427 Muhammad MS., Magaji RA., Mohammed A., Isa A-S., Magaji MG. 2017. Effect of resveratrol

428

429 and environmental enrichment on biomarkers of oxidative stress in young healthy mice.

Metabolic Brain Disease 32:163-170. DOI: 10.1007/s11011-016-9891-1.

430 Ng F., Berk M., Dean O., Bush AI. 2008. Oxidative stress in psychiatric disorders: evidence base 431 and therapeutic implications. The International Journal of Neuropsychopharmacology 11:851-

432 876. DOI: 10.1017/S1461145707008401.

433 Nilsson M., Perfilieva E., Johansson U., Orwar O., Eriksson PS. 1999. Enriched environment

434 increases neurogenesis in the adult rat dentate gyrus and improves spatial memory. Journal of

435 Neurobiology 39:569-578. 
436 Nithianantharajah J., Hannan AJ. 2006. Enriched environments, experience-dependent plasticity 437 and disorders of the nervous system. Nature Reviews Neuroscience 7:697-709. DOI:

438 10.1038/nrn1970.

439 Otte C., Gold SM., Penninx BW., Pariante CM., Etkin A., Fava M., Mohr DC., Schatzberg AF. 440 2016. Major depressive disorder. Nature Reviews. Disease Primers 2:16065. DOI:

$441 \quad 10.1038 /$ nrdp.2016.65.

442 Pacher P., Beckman J s., Liaudet L. 2007. Nitric Oxide and Peroxynitrite in Health and Disease.

443

444

445

Physiological reviews 87:315-424. DOI: 10.1152/physrev.00029.2006.

446

447

448

449

450

451

452

453

454

455

456 Piato ÂL., Capiotti KM., Tamborski AR., Oses JP., Barcellos LJG., Bogo MR., Lara DR., Vianna MR., Bonan CD. 2011. Unpredictable chronic stress model in zebrafish (Danio rerio): behavioral and physiological responses. Progress in Neuro-Psychopharmacology \& Biological Psychiatry 35:561-567. DOI: 10.1016/j.pnpbp.2010.12.018.

Poprac P., Jomova K., Simunkova M., Kollar V., Rhodes CJ., Valko M. 2017. Targeting Free Radicals in Oxidative Stress-Related Human Diseases. Trends in Pharmacological Sciences 38:592-607. DOI: 10.1016/j.tips.2017.04.005.

van Praag H., Kempermann G., Gage FH. 2000. Neural consequences of environmental enrichment. Nature Reviews. Neuroscience 1:191-198. DOI: 10.1038/35044558.

Rambo CL., Mocelin R., Marcon M., Villanova D., Koakoski G., de Abreu MS., Oliveira TA., Barcellos LJG., Piato AL., Bonan CD. 2017. Gender differences in aggression and cortisol levels in zebrafish subjected to unpredictable chronic stress. Physiology \& Behavior 171:50-54. DOI: 10.1016/j.physbeh.2016.12.032.

457 Sale A., Maya Vetencourt JF., Medini P., Cenni MC., Baroncelli L., De Pasquale R., Maffei L. 458 2007. Environmental enrichment in adulthood promotes amblyopia recovery through a reduction 459 of intracortical inhibition. Nature Neuroscience 10:679-681. DOI: 10.1038/nn1899.

460 Sherwin CM. 2004. The influences of standard laboratory cages on rodents and the validity of 461 research data. Animal Welfare 13:9-15.

462 Shilpa BM., Bhagya V., Harish G., Srinivas Bharath MM., Shankaranarayana Rao BS. 2017. 463 Environmental enrichment ameliorates chronic immobilisation stress-induced spatial learning 464 deficits and restores the expression of BDNF, VEGF, GFAP and glucocorticoid receptors.

465 Progress in Neuro-Psychopharmacology \& Biological Psychiatry 76:88-100. DOI:

466 10.1016/j.pnpbp.2017.02.025.

467 Sies H., Berndt C., Jones DP. 2017. Oxidative Stress. Annual Review of Biochemistry 86:715468 748. DOI: 10.1146/annurev-biochem-061516-045037.

469 Song C., Liu B-P., Zhang Y-P., Peng Z., Wang J., Collier AD., Echevarria DJ., Savelieva KV., 470 Lawrence RF., Rex CS., Meshalkina DA., Kalueff AV. 2017. Modeling consequences of 471 prolonged strong unpredictable stress in zebrafish: Complex effects on behavior and physiology.

472 Progress in Neuro-Psychopharmacology \& Biological Psychiatry. DOI:

473 10.1016/j.pnpbp.2017.08.021.

474 Sun XR., Zhang H., Zhao HT., Ji MH., Li HH., Wu J., Li KY., Yang JJ. 2016. Amelioration of 475 oxidative stress-induced phenotype loss of parvalbumin interneurons might contribute to the 476 beneficial effects of environmental enrichment in a rat model of post-traumatic stress disorder. 477 Behavioural Brain Research 312:84-92. DOI: 10.1016/j.bbr.2016.06.016.

478 Sztainberg Y., Chen A. 2010. An environmental enrichment model for mice. Nature Protocols 479 5:1535-1539. DOI: 10.1038/nprot.2010.114.

480 Tanti A., Westphal W-P., Girault V., Brizard B., Devers S., Leguisquet A-M., Surget A., 481 Belzung C. 2013. Region-dependent and stage-specific effects of stress, environmental 
482 enrichment, and antidepressant treatment on hippocampal neurogenesis. Hippocampus 23:797483 811. DOI: 10.1002/hipo.22134.

484 Toth LA., Kregel K., Leon L., Musch TI. 2011. Environmental Enrichment of Laboratory

485 Rodents: The Answer Depends on the Question. Comparative Medicine 61:314-321.

486 Young D., Lawlor PA., Leone P., Dragunow M., During MJ. 1999. Environmental enrichment

487 inhibits spontaneous apoptosis, prevents seizures and is neuroprotective. Nature Medicine 5:448488 453. DOI: $10.1038 / 7449$.

489 Zorov DB., Juhaszova M., Sollott SJ. 2014. Mitochondrial Reactive Oxygen Species (ROS) and 490 ROS-Induced ROS Release. Physiological Reviews 94:909-950. DOI:

491 10.1152/physrev.00026.2013.

492 
Figure 1

Housing conditions. A) Barren tank; B) Enriched environment tank. Image credit/source: ( $A$ and $B$ ) Matheus Marcon.

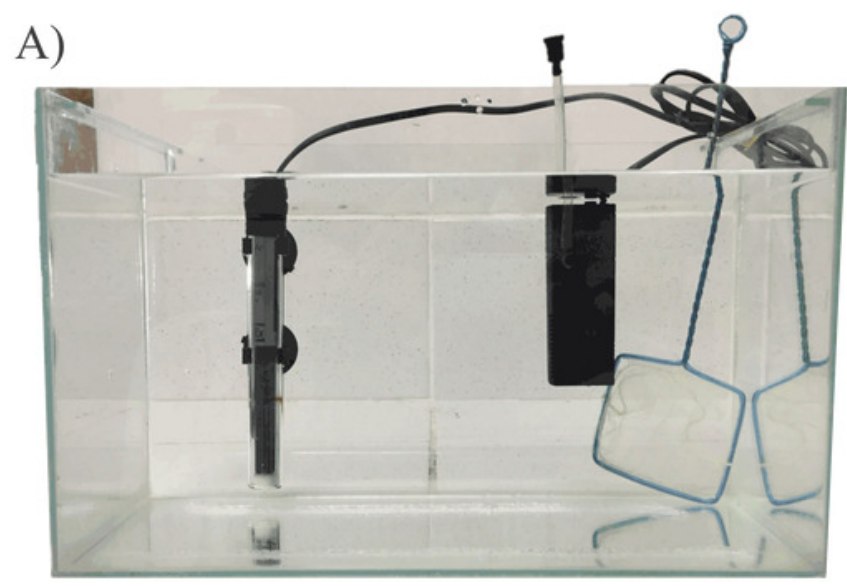

B)

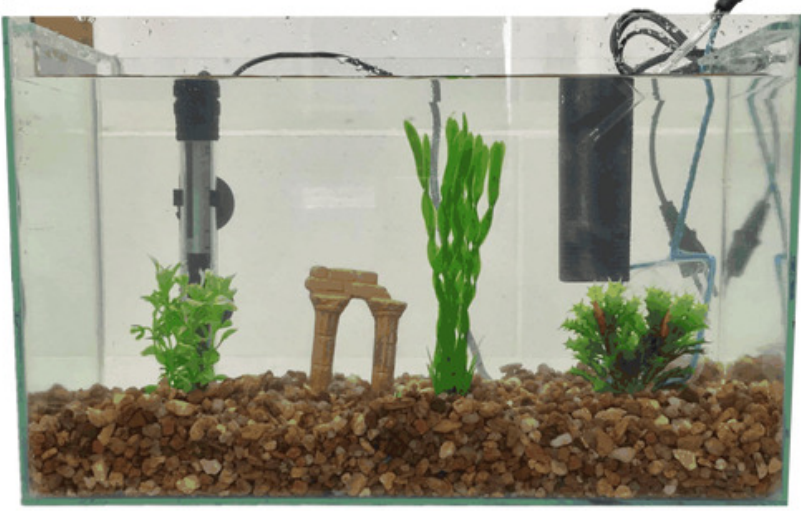




\section{Figure 2 (on next page)}

Experimental design.

The fish were housed in barren tank (BARREN) or enriched environment tank (EE) for 21 or 28 days. In the last 7 days of the experimental protocol, they were submitted to the unpredictable chronic stress (UCS) protocol or remained unchanged (Control). The day after the last stressor, at 08:00 a.m., the fish were euthanized for biochemical analyzes of oxidative stress (the brain was used for the dosage of the brain was used for the dosage of lipid peroxidation (TBARS), reactive oxygen species (ROS) levels, non-protein thiol (NPSH) and total thiol (SH) levels, superoxide dismutase (SOD) and catalase (CAT) activities. 
PeerJ Exposure periodu(days) ${ }^{\text {viewed }}$

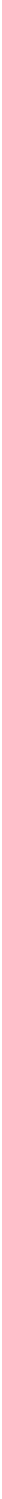


Figure 3

Effects of enriched environment for 21 (EE 21) or 28 days (EE 28) on biochemical parameters associated with oxidative stress in zebrafish brain submitted to unpredictable chronic stress (S+) or not (S-).

Lipid peroxidation (TBARS) and reactive oxygen species (ROS) levels. BARREN: barren tank. Data are expressed as a mean + S.E.M. $n=5$. Two-way ANOVA/Tukey.

A

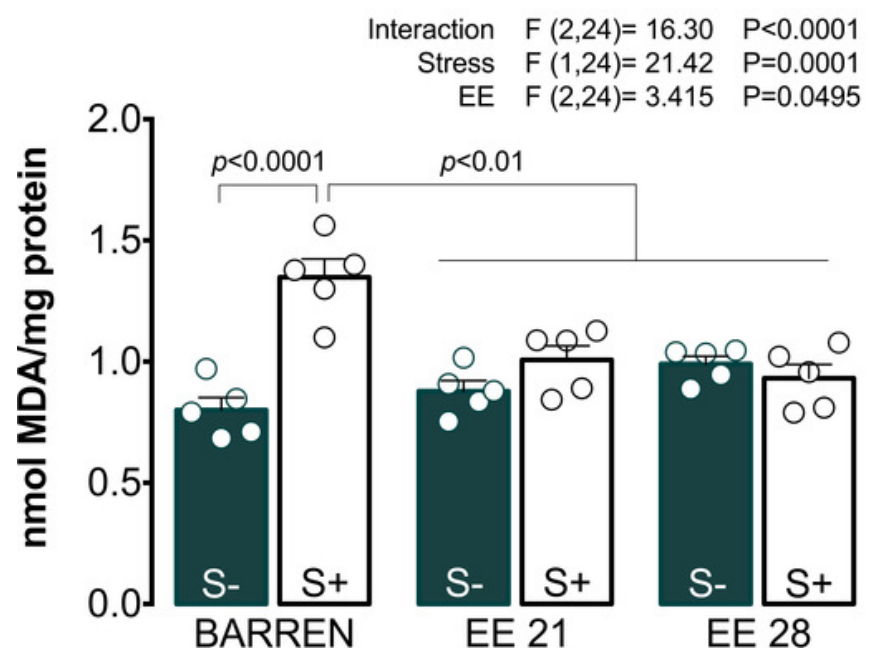

B

ROS levels

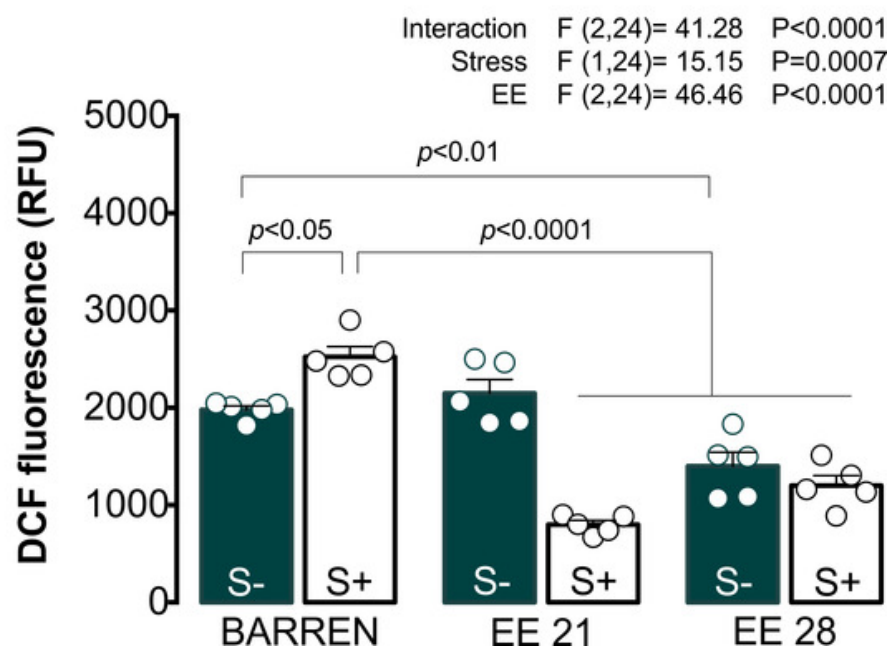


Figure 4

Effects of enriched environment for 21 (EE 21) or 28 days (EE 28) on antioxidant mechanisms in zebrafish brain submitted to unpredictable chronic stress (S+) or no (S-). Non-protein thiols (NPSH) and Total thiol (SH) levels.

BARREN: barren tank. Data are expressed as a mean + S.E.M. $n=5$. Two-way ANOVA/Tukey.

A NPSH levels

Interaction $\quad F(2,24)=3.942 \quad P=0.0331$

Stress $F(1,24)=4.663 \quad P=0.0410$

EE $F(2,24)=0.282 \quad P=0.7566$

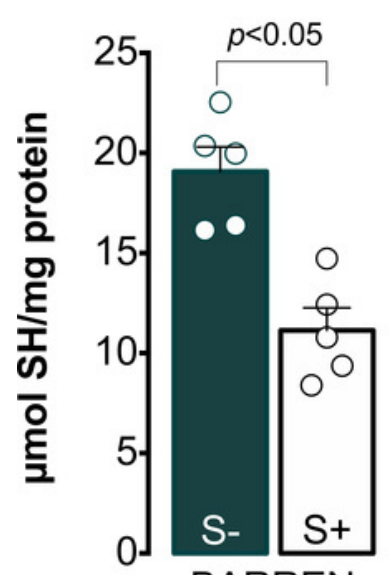

BARREN

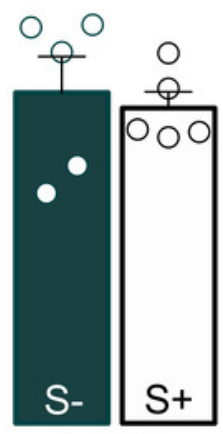

EE 21

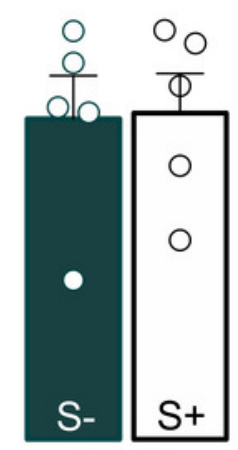

EE 28

B SH total levels

Interaction $\quad F(2,24)=3.926 \quad P=0.0335$

Stress $F(1,24)=3.109 \quad P=0.0906$ EE $F(2,24)=0.474 \quad P=0.6279$

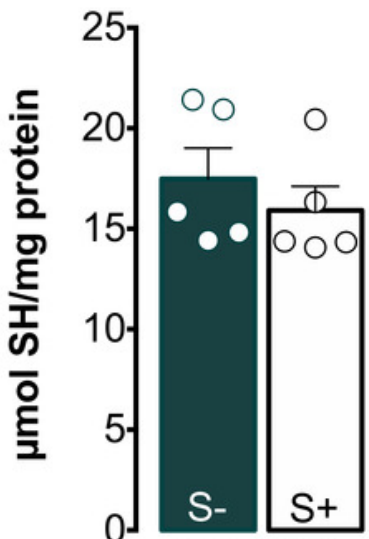

BARREN

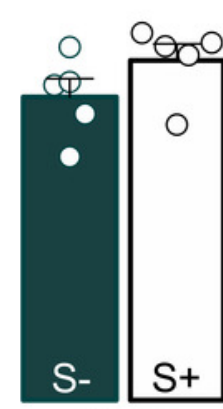

EE 21

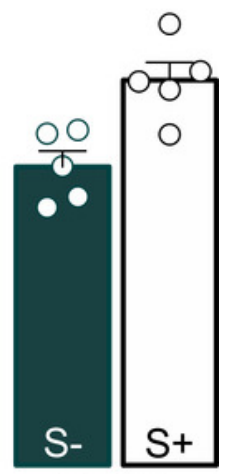

EE 28 
Figure 5

Effects of enriched environment for 21 (EE 21) or 28 days (EE 28) on antioxidant mechanisms in zebrafish brain submitted to unpredictable chronic stress (S+) or no (S-).

Superoxide dismutase (SOD) and Catalase (CAT) activity. BARREN: barren tank. Data are expressed as a mean + S.E.M. $n=5$. Two-way ANOVA/Tukey.

A

\section{SOD activity}

Interaction $\quad F(2,24)=3.598 \quad P=0.0430$

Stress $F(1,24)=42.46 \quad P<0.0001$

EE $F(2,24)=3.408 \quad P=0.0498$

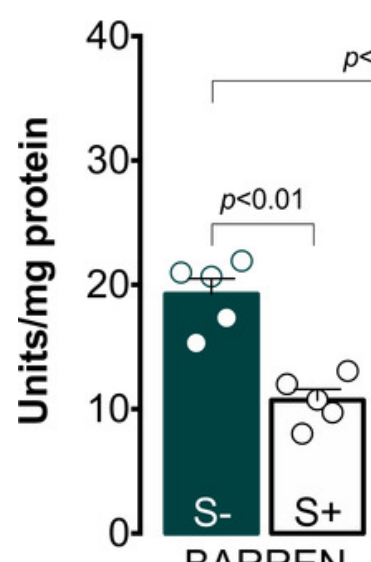

BARREN

$p<0.05$

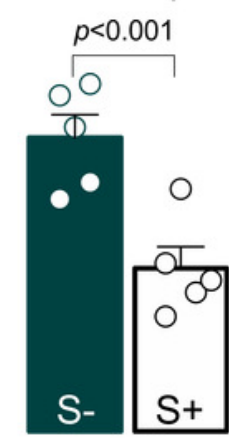

EE 21

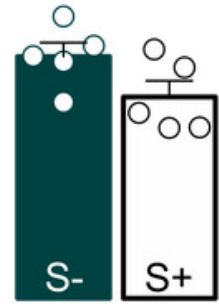

EE 28
B

CAT activity
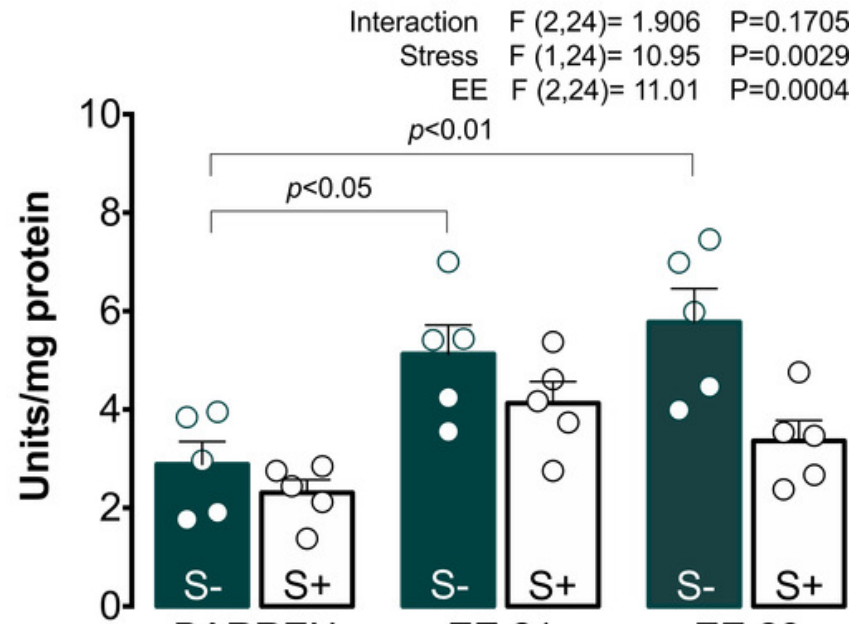

BARREN EE 21

EE 28 